\title{
Retour à l'utopie sans socialisme : La Ville noire de George Sand
}

\section{Agnese Silvestri}

\section{Q OpenEdition}

1 Journals

\section{Édition électronique}

URL : http://journals.openedition.org/rief/220

DOI : $10.4000 /$ rief.220

ISSN : 2240-7456

\section{Éditeur}

Seminario di filologia francese

\section{Référence électronique}

Agnese Silvestri, «Retour à l'utopie sans socialisme : La Ville noire de George Sand », Revue italienne d'études françaises [En ligne], 3 | 2013, mis en ligne le 15 décembre 2013, consulté le 10 décembre 2020. URL : http://journals.openedition.org/rief/220 ; DOI : https://doi.org/10.4000/rief.220

Ce document a été généré automatiquement le 10 décembre 2020.

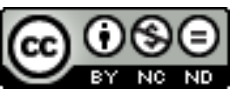

Les contenus de la RIEF sont mis à disposition selon les termes de la Licence Creative Commons Attribution - Pas d'Utilisation Commerciale - Pas de Modification 4.0 International. 


\title{
Retour à l'utopie sans socialisme : La Ville noire de George Sand
}

\author{
Agnese Silvestri
}

Qu'en est-il, à l'époque du Second Empire, des perspectives progressistes des travailleurs pour une écrivaine comme George Sand, qui s'était investie dans la révolution de 1848 et qui n'avait pas hésité à accueillir dans ses œuvres les idées du socialisme utopique ? La réponse n'est pas simple car si Sand garde un cadre de valeurs tout compte fait stable, sa pensée politique n'en évolue pas moins. L'idée de l'égalité entre les hommes, celle d'un progrès indéfectible vers l'harmonie sociale, l'intérêt généreux pour le sort des plus démunis, se retrouvent certainement jusqu'à la fin de sa vie. Pourtant, sa méfiance envers l'enrichissement individuel s'estompe graduellement; dans ses « romans mondains » des années 1860 , elle se montre sensible aux " principes de la morale courante ${ }^{1}$; et dans sa toute dernière production, l'idée socialiste d'une propriété commune, garantie de justice sociale, cède le pas aux principes de la démocratie libérale ${ }^{2}$. Rares, ses textes des années 1860 à soubassement théorique témoignent des modifications de sa pensée politique ainsi que plus d'une réserve à l'égard des ouvriers. Prendre en compte ces changements est d'autant plus nécessaire pour une écrivaine qu'on a dès lors vite et volontiers réduite à l'image de "Bonne Dame de Nohant», toute compassion et sains principes bourgeois affichés. De ce point de vue, La Ville noire (1861), roman consacré aux ouvriers des usines de coutellerie et de papeterie installées le long de la Durolle, constitue un point d'observation privilégié pour évaluer la réflexion sociale et politique sandienne. Les liens thématiques de cette œuvre avec les narrations des années 1840, sa représentation du progrès industriel, l'inauguration d'un type nouveau d'héroïne, Tonine, douée d'une capacité d'intervention sur le réel jusqu'alors inconnue dans l'univers sandien, la création d'un personnage d'ouvrier réformateur social et fou, Audebert, par le biais duquel Sand semble régler ses comptes avec ses certitudes d'autrefois, donnent tout son intérêt à sa fiction littéraire de cette époque. L'analyse des personnages, de leurs parcours, du monde utopique dans lequel ils interviennent, ne saurait se faire sans mettre en relation l'invention littéraire avec les positions politiques, littéraires et existentielles de Sand, ainsi qu'avec ses efforts pour les analyser et les préciser. 


\section{Mise en contexte}

Lorsque Sand compose La Ville noire, du 4 au 28 janvier 1860, sa position envers le Second Empire est désormais claire tout comme sa prise de distance par rapport aux socialistes républicains qui, contrairement à elle, n'acceptent pas le résultat du plébiscite ${ }^{3}$. Notamment, Sand n'a pas choisi l'opposition ouverte, ce que ne lui pardonnent pas ceux qui sont restés adversaires inébranlables du coup d'État. Elle est intervenue auprès de Louis-Napoléon Bonaparte sans renier ses principes et dans le but d'adoucir le sort des amis pourchassés, mais elle n'en a pas moins cherché à pactiser avec le nouveau régime. Elle vit dans ce cadre-là non sans amertume, en déplorant la censure, en se retranchant "dans un cercle chaque jour plus étroit $»^{4}$. Pourtant, elle cultive son amitié avec le cousin de l'Empereur et, si elle regrette le «degré de liberté » atteint par «la critique de la société réelle et le rêve d'une société idéale » qui lui avait permis d'écrire en 1845 Le Péché de Monsieur Antoine ${ }^{5}$, elle a désormais accepté de bien mesurer ses mots. Un peu plus tard, elle parlera de "soumission religieuse à la loi dans tous nos actes extérieurs " ${ }^{6}$, et jugera la philosophie de L. Brothier comme un rempart contre le pessimisme, étant donné que tout moment de recul qui succède aux élans des révolutions n'est qu'une phase nécessaire, comprise dans la «continuité » du progrès humain7. À Hugo qui, en 1859, dédaigne l'amnistie, Sand oppose, froissée, sa « résignation »: «Je vis dans l'idéal, cela c'est vrai, mais j'y vis seule, et voyant les autres heureux sans cela autour de moi, je suis forcée de ne pas les troubler de rêves importuns. Donc je ne jette point de flammes et je vis à l'état de lanterne sourde $»^{8}$.

3 En effet, loin de faire scandale, La Ville noire passe presque inaperçu. Conséquence de la censure qui pèse sur la presse, d'après certains ${ }^{9}$, marque de réprobation pour un sujet "bas » et "grossier», selon d'autres ${ }^{10}$. Buloz lui-même, qui à l'époque d'Horace (1842) avait rompu ses relations avec l'écrivaine à cause des propos politiques contenus dans le récit, ne trouve rien à redire cette fois ${ }^{11}$. On ne rendrait pourtant pas justice à ce roman, si l'on se limitait à souligner sa prudence par rapport aux romans inspirés par les doctrines égalitaires. Certes, dans $L a$ Ville noire, aucun personnage ne discute les théories des saint-simoniens et des fouriéristes, contrairement à ce qui arrive dans Horace, dans Le Meunier d'Angibault, dans Le Péché. Pourtant, plus d'un indice révèle la parenté de La Ville noire avec la production précédant la révolution de 1848. Il ne s'agit pas seulement du sujet, ce retour sur le monde du travail salarié à vingt ans de distance du Compagnon du Tour de France (1841). Le fait, isolé, que l'élan narratif emprunte les formes du chant à structure anaphorique - l'hyménée final qui célèbre le lien des deux héros, Tonine l'ouvrière plieuse et Sept-Épées -, comme à l'époque de la Comtesse de Rudolstadt (1844) et de sa "Ballade à la bonne déesse de la Pauvreté », ne suffit pas non plus. Mais il est peutêtre plus décisif que le récit, dans son ensemble, se lise de nouveau comme une « parabole » exemplaire de ce qui pourrait ou devrait être le sort du prolétariat dans un monde meilleur ${ }^{12}$. Loin de se replier sur l'acceptation résignée de l'injustice sociale, Sand dessine pour les travailleurs de la "ville noire » - la ville où l'on peine à la forge, noircie et insalubre, opposée à la "ville haute » des bourgeois, qui étale, elle, sa richesse et sa beauté au soleil - un changement positif qui concerne l'urbanisation, les conditions de travail, l'organisation sociale. Autre facteur significatif, le ton optimiste du récit, qui se distingue fort de l'« écriture de la mélancolie ${ }^{13}$ qu'on trouve dans les romans du Second Empire où, pour se tenir à des créations proches de La Ville noire, perce ci et là la nostalgie 
pour "l'ère nouvelle des relations" sociales, un moment inaugurée et tout de suite refermée en $1848^{14}$; ou pour les élans idéaux des Romantiques, suffoqués par «la soif d'argent, [le] plaisir sans idéal, [les] ambitions sans frein » de l'âge contemporain ${ }^{15}$. Pas de regrets nostalgiques, dans $L a$ Ville noire, mais la narration d'un nouveau modèle de développement dont la construction est confiée à une femme, l'ouvrière Tonine.

La Ville noire constitue bien un retour en terre d'utopie progressiste. Ce qui ne veut pas dire un simple retour en arrière, car ce roman présente quelques nuances presque rancunières face à l'échec du socialisme utopique incapable de «trouver le remède à la misère » $(V N, 104)$ et montre bien des différences par rapport aux solutions envisagées autrefois. Pourtant, en l'écrivant, Sand sentait qu'elle avait encore « dans le cœur quelque vérité à dire et à redire $»^{16}$. Ce qui peut expliquer la place que, parmi les créations des années 1858-1860, cette œuvre occupe dans sa correspondance. La romancière investit en effet La Ville noire d'une volonté de rachat artistique qui engage profondément sa satisfaction personnelle. Elle affirme l'avoir écrit avec "soin», avec "amour», en espérant produire une " perle $»^{17}$. À Aucante, elle explique : «[C]omme je fais cela pour me bien remonter sur l'eau, littérairement parlant, il faudra penser à l'effet plus qu'au profit ${ }^{18}$. Ce besoin de "remonter " s'explique, en partie, à la lumière des attaques assez violentes que Sand a endurées en 1858 après la publication d'Histoire de ma vie et, en 1859, à la sortie d'Elle et Lui, dans la Revue des Deux Mondes, roman autobiographique sur sa relation avec Musset qui venait de disparaître. P. Salomon souligne donc la nécessité pour Sand d'écrire des œuvres « d'une moralité inattaquable » et La Ville noire remplirait selon lui la tâche ${ }^{19}$. Pourtant, lorsque Sand révèle à son fils Maurice : «Il faut que ce soit très soigné pour faire passer bien la tentative d'une nouvelle manière $»^{20}$, quand elle lui avoue sa peine d'écrivaine - "Je suis occupée de refaire une partie de mon chef-d'œuvre (espéré) de la Ville noire, et de refaire toute la fin dont je n'étais pas bien contente ${ }^{21}$ - son souci esthétique, et le goût qu'elle prend à l'écriture, semble largement dépasser l'aspiration à corriger son image publique de femme à scandales.

5 Car elle cherche à atteindre la perfection artistique en peignant les prolétaires de l'industrie auvergnate. De plus, contrairement à la diversité de la représentation sociale présente d'habitude dans ses romans, elle s'efforce de poser presque exclusivement, au centre de ce roman de formation, le point de vue des travailleurs. L'écrivaine sent son imagination renouer avec les temps optimistes et politiquement orientés de l'écriture du Compagnon. Elle en est consciente et l'avoue à Perdiguier, son mentor en compagnonnage, lui expliquant que, tout en se tenant à distance "des détails réels et positifs de la vie ouvrière", le roman propose "un résumé poétique et moral des émotions, des sentiments, des passions, et des aspirations qui peuvent fleurir au sein du travail ${ }^{22}$. Comme aux temps de la création de Pierre Huguenin, Sand revendique donc sa faculté de regarder le présent du peuple tout en apercevant son avenir idéa ${ }^{23}$, pour proposer aux prolétaires des modèles de leur progrès. Les temps étant changés, toutefois, elle expérimente un nouveau modèle littéraire qui, avec ses « dialogues courts et simplement résumés par des gens à idées peu compliquées ${ }^{24}$, renonce au dialogisme recherché d'antan comme aux longues tirades didactiques. Le côté plus proprement idéologique est confié aux actions des personnages et à leur résultat. La censure ne l'aurait pas permis autrement, certes, mais ce choix esthétique n'est pas sans lien avec tout ce qui, des déceptions d'après 1848 aux modifications de la pensée politique de l'écrivaine, lui empêchent de "prêcher ", comme autrefois, l'évangile socialiste. Le personnage de Tonine, moins porteur de discours que d'actions, ainsi que celui d'Audebert qui sonne le 
glas du prolétaire prophète de révolutions égalitaires, on le verra, en constituent en ce sens une preuve ultérieure.

6 La difficulté d'analyse de La Ville noire, où le réseau des significations se charge d'ambiguïté, dépend des glissements idéologiques sandiens dans l'appréciation de la réalité sociale : rien n'est ouvertement renié des croyances d'antan mais rien n'est plus comme autrefois. Deux textes à portée théorique encadrent idéalement ce roman. D'une part, les deux longues lettres à Rodrigues de 1863, où Sand définit ses idées politiques. De ces textes complexes, on ne retiendra que Sand confirme comme fondamentale l'égalité des hommes et " insupportable l'injustice sociale $~^{25}$. Mais le socialisme y est défini assez vaguement comme " une science qui a pour but le progrès de l'humanité au moyen des institutions sociales ", sans qu'il soit plus question de partage des propriétés ${ }^{26}$. À sa place, on trouve "l'association libre $»^{27}$ et une tentative de compromis équilibré entre les besoins de la société et ceux de l'individu. Sand corrige le précepte saint-simonien «à chacun selon sa capacité et à chacun selon son œuvre », par la leçon de Blanc «à chacun suivant ses besoins", mais identifie ces derniers avec « ce que la loi et la société savent nécessaires à l'homme : l'instruction et le développement $»^{28}$. Sur le sens à donner à ce dernier mot, le discours de La Ville noire est précieux. Peu de doutes que l'industrie ne soit désormais considérée par Sand comme un des instruments. Le coutelier Gaucher, bien que conscient d'être " condamné à faire toujours la même chose [sa] vie durant ", est tout heureux des possibilités d'emploi que les usines de la « ville noire » offrent : « huit mille hommes chaque jour assurés du lendemain et pouvant ainsi, par le travail, aller du jeune âge à la mort sans trop de misère et de souci » (VN, 58-59). Cette appréciation du travail ne cache pas sa dureté - l'« enfer de la forge » $(V N, 54)$, le " gouffre où nous voici attelés du matin au soir » $(V N, 56)$, « tous ces bruits qui fendent la tête » $(V N, 57)$ - mais présente l'industrie comme une possibilité de vie digne où le problème de l'exploitation au profit de peu de gens n'a plus guère de place. Ce qui est bien loin du cri d'indignation qui résonnait dans Le Péché, où le marginal Jappeloup dénonçait le désert économique, social et politique créé par le système de pouvoir de l'usine de Cardonnet sous couvert d'apporter du progrès et du travail pour tous.

7 Un tel retournement est le fruit de la prise en compte de la part de Sand de l'essor industriel, ainsi que de sa confiance accrue dans le pouvoir du progrès technologique « les machines feront les choses malpropres [...]. L'homme doit cesser de faire l'ouvrage de la bête $»^{29}-$, déjà promu par le saint-simonisme. En reconnaissant que l'avenir économique repose sur l'industrie, Sand s'évertue à en imaginer un type positif. Ce qui ne va pas, toutefois, sans idéalisation du système capitaliste. Déjà le bourgeois Narcisse, dans le roman éponyme de $1859^{30}$, n'attend plus de l'industrie que «le bienfait de l'aisance » pour « le pauvre monde » de son village $(N, 34)$, tandis que les capitalistes qui investissent dans son projet sont pleins de «bonnes intentions" et prêts à soutenir la création "d'établissements de charité ", l'infirmerie pour les ouvriers et une école pour leurs enfants $(N, 37)$. Par rapport à ce roman, La Ville noire propose pourtant un véritable système intégré et autonome, où l'usine gérée par l'ancienne plieuse Tonine génère tout ce qui peut rendre la vie des travailleurs plus humaine, plus heureuse, plus complète. Il s'agit moins de charité que d'une formule économique investissant les profits créés par l'usine dans l'amélioration de la vie des travailleurs - j'y reviendrai.

8 L'autre écrit dont il faut tenir compte est daté de mars 1860, mais Sand ne se décide à le publier qu'en 1871, après la Commune, ce qui soulève plus d'une question. Il s'agit du deuxième feuilleton paru dans le Temps, le 5 septembre, sans titre, et consacré à 
l'évaluation de l'avenir socio-politique de la nation ${ }^{31}$. Tout en affirmant «croi[re] à un immense avenir pour le peuple français", Sand se livre à des jugements très durs concernant ce dernier. Le peuple lui apparaît "mystérieux", "redoutable», «lancé comme la bourgeoisie dans la vie effrénée », ayant « pris le goût du vice » (IS, 55-59). On retrouve ici la nostalgie dont le roman avait fait abstraction: aux "poètes, [aux] économistes, [aux] apôtres, élite naïve, ignorante, mais parfois inspirée, et qui travaillait véritablement à s'instruire » (IS, 56), s'est substitué un ouvrier " poseur et prétentieux ", qui aime s'écouter parler (IS, 60). L'idéal représenté par le personnage de Pierre Huguenin, "portrai[t] flatt[é]» mais non "pur[e] chimèr[e]» (IS, 57), ressemble à une occasion historique ratée. On peine à croire que ce texte, composé à Paris, suit de si près (seulement un mois) l'écriture de La Ville noire, tant est grande la distance entre le regret d'un Pierre Huguenin perdu et l'image d'une Tonine, exemple positif, qui en recueille l'héritage. Cet article est le signe d'une distanciation par rapport aux voies d'émancipation choisies par le prolétariat historique et, peut-être, d'une difficulté de Sand à se confronter à l'ouvrier parisien. Le refus de la pensée socialiste de la deuxième moitié du siècle n'aide pas l'écrivaine. Il est vrai qu'en 1871 Sand se défendra publiquement, et passionnément, de toute interprétation réactionnaire de son article ${ }^{32}$. Pourtant, lu à la lumière du roman, on a l'impression d'un mauvais rêve qu'elle arrive encore à exorciser efficacement par la création littéraire, mais qui la guette. Aucun danger ne vient des travailleurs de La Ville noire - ils ne font de mal qu'à eux-mêmes -, aucun d'eux n'est un débauché. Pourtant, ils sont cruellement punis de leur ambition à abandonner la condition ouvrière et de leurs rêves, généreux mais prétentieux, de pouvoir trouver un système théorique en mesure d'assurer la justice sociale.

\section{De l'ascension sociale de l'ouvrier}

$9 \quad$ L'inquiétude douloureuse qui tourmente Sept-Épées sur laquelle s'ouvre La Ville noire est d'une autre nature que le chagrin de son frère aîné Huguenin. Le menuisier était intérieurement travaillé par «le sentiment d'une destinée commune à tous les travailleurs ", par la volonté de surmonter leurs divisions et d'unir leurs efforts contre l'exploitation ${ }^{33}$; l'armurier en est à décider s'il vaut la peine de renoncer à l'amour et d'endurer "une vingtaine d'années de courage et d'entêtement» pour sortir de l'« enfer » de la ville basse ${ }^{34}$ et monter au « paradis » bourgeois de la ville haute $(V N, 56)$. À la perspective collective s'est substituée la visée individuelle. Mais tout n'est pas dit en reconnaissant que le récit se charge de montrer toute l'inanité de cette dernière approche, car ce n'est pas sans ambiguïté qu'on pose le problème de l'ascension sociale des ouvriers. Sand laisse bien sentir au lecteur la légitimité de l'aspiration de Sept-Épées à se soustraire à « un travail de machine », à un « esclavage » (VN, 87). Le roman condamne toutefois à la défaite, économique ou morale, quiconque tente de réussir individuellement. C'est le cas de personnages mineurs dont on raconte l'histoire (la sœur de Tonine); c'est le cas de quelques personnages principaux : Audebert, Sept-Épées. Ce dernier, qui a de l'esprit et de l'ambition, rêve de «monter une fabrique à [son] compte » $(V N, 55)$ mais, au mieux de ses affaires, doit reconnaître « qu'il n'achèterait jamais une maison peinte et un parc fleuri dans la ville haute » $(V N, 199)$. Faire fortune sans moyens se révèle illusoire - «avec peu on fait peu» $(V N, 198)$-, tandis que le chemin de la renommée pour avoir découvert un nouveau mécanisme reste interdit à l'ouvrier qui n'a pas l'instruction nécessaire. À la recherche d'un enrichissement subit ou, au moins, de 
gloire, Sept-Épées apprend à ses dépens ce que peut être une attitude humble qui confine à la résignation : « les vaines sensibilités, les poignantes aspirations doivent s'émousser et faire place à une espèce de fatalisme robuste » $(V N, 208)$, songe-t-il, presqu'au terme de son parcours, avant de découvrir que son usine a été emportée par la Durolle et qu'il a tout perdu. Le roman décrit donc le « démon de l'ambition » (VN, 87), «l'amour du gain qui pousse les ambitieux jusque dans les précipices » $(V N, 215)$, et même l'orgueil insensé de l'ouvrier qui voudrait se signaler par son génie créatif.

Il y a une part de réalisme dans cette estimation des possibilités réelles de changement de statut social. On peut pourtant se demander s'il n'y a pas une espèce d'interdit qui pèse sur la mobilité spatiale et sociale des ouvriers. Une des injonctions de l'hyménée final chante : «Nobles enfants du travail, ne quittez jamais la ville noire !» $(V N, 254)$, mais Sand ne nous donne plus à voir, comme autrefois, la bassesse morale des bourgeois, l'égoïsme féroce de ceux qui ne vivent que pour l'argent, la détermination autoritaire vouée à l'exploitation des autres ${ }^{35}$. Non qu'elle les approuve, les seuls bourgeois qui ne soient pas de pâles silhouettes, $M$. Anthime et son enfant docteur, sont sensibles et très généreux, l'un, «homme très bon et très savant » $(V N, 106)$, l'autre, " ami des pauvres comme son père!» $(V N, 187)$. En outre, le personnage qui se caractérise par le rejet orgueilleux de la ville haute, le père Laguerre, est un homme âgé, confiné dans un espace urbain et mental étriqué (" Travailleur austère, cœur dévoué, cerveau étroit », VN, 71), ce qui, avec son immobilisme, le place du côté des exemples à ne pas suivre. Enfin, et surtout, il reste quelque chose du respect admiratif avec lequel Gaucher regarde les "gens riches » de la ville haute qui, d'ouvriers qu'ils étaient ou qu'étaient leurs parents, ont mérité par le travail leur réussite sociale : ils « ont bien gagné ce qu'ils sont » $(V N, 56)$, assure-t-il. Ce changement d'attitude envers la bourgeoisie se fonde sur la valeur du travail. Sand l'avait déjà formulé, en 1855, en affirmant que le bourgeois qui travaille honnêtement, avec des profits modestes, s'abstenant de spéculation boursière et industrielle, «est le peuple ${ }^{36}$. Dans La Ville noire, les bourgeois ne méprisent pas les travailleurs de la ville basse, soutient Gaucher, car ils « regardent avec orgueil les fumées et les tonnerres de la ville basse monter dans les airs, comme un cantique et un encens, en l'honneur de celui qui les a fait grandir et prospérer» $(V N, 59)$. L'horreur de Tonine à « devenir dame dans la ville haute » où elle « serai[t] moquée comme [s]a pauvre sœur l'a été » $(V N, 185)$ laisse pourtant penser que ce respect n'est possible qu'à condition « de ne pas sortir de [s]on état » $(V N, 81)$, comme Tonine refuse en effet de le faire.

11 D'un autre côté, il est indéniable que le roman cherche à valoriser l'identité ouvrière ${ }^{37}$. Sand, qui pendant ses excursions à Thiers avait trouvé « pittoresques » les « usines noires, amas de planches d'où sortent des bruits incessants $"^{38}$, a voulu éviter un regard dévalorisant sur la ville basse. C'est la raison pour laquelle même les personnages qui aspirent ou aspiraient à un changement de leur mode de vie, la regardent avec admiration : " tout cela me danse devant les yeux et me chante dans les oreilles ", affirme Gaucher en décrivant les toits noirs, les hangars, les passerelles, les fracas, et même les "enfants barbouillés de suie et de limaille ( $V N, 58)$. Empruntant le regard de la romancière, Sept-Épées regarde ébloui «le tableau bizarre et animé de la ville noire, pittoresque décor de fabriques enfumées et de cascades étincelantes, amas de charbons et de diamants, sanctuaire de travail ardent au sein d'une nature âpre et sublime » $(V N, 155)$. En tout cas, c'est ce « cantique » du travail dont parle Gaucher au début que Sand donne à entendre à la fin du roman, avec l'hyménée dont «l'ouverture » musicale $(V N, 247)$ n'est autre que le bruit des roues, des marteaux et des cascades, savamment actionnés pour 
l'occasion. Et ce sont les « saintes voix du travail » $(V N, 254)$ accompagnant l'épithalame qui conduisent les bourgeois de la ville haute en route pour rendre respectueusement hommage à Tonine qui se marie dans la ville basse: «le petit et le gros commerce, la jeune et la vieille bourgeoisie de la ville haute, avec la musique en tête et le peuple en queue, [...] descendaient vers la rivière » $(\mathrm{VN}, 254)$. Ce mouvement collectif de descente correspond au fait que, dans le récit, toute ascension individuelle se trouve systématiquement frustrée - à l'exception de celle, accidentelle, de Tonine, qui ne l'avait pas cherchée et qui d'ailleurs l'interprète comme un service à ses concitoyens. La voie à suivre n'est pas le changement de statut social de l'individu, mais au contraire le progrès collectif des conditions de vie - celui réalisé par Tonine, qui renforce l'identité et la dignité ouvrières. Ce qui rend possible le dialogue avec la bourgeoisie sur un pied d'égalité, au moins le temps d'une fête : "les deux villes rivales, mais toujours sœurs » peuvent enfin « se mêler cordialement dans une fête improvisée » $(V N, 255)$.

\section{La fin, peu honorable, du prolétaire prophète}

L'histoire de Sept-Épées et celle d'Audebert s'entrecroisent. Les deux se sont cru « appelé [s] à de hautes destinées " et ont "subi la maladie du siècle.» $(V N, 202)$. Parmi les personnages qui refusent le sort malheureux du travailleur, Audebert est le seul qui, totalement désintéressé, passe d'un projet individuel (adopter un orphelin pauvre) à une perspective collective ("trouver un remède à la misère ", VN, 104). Comme Sand le transforme en poète, fou et ridicule de surcroît, son cas interpelle le lecteur de la façon la plus troublante. Audebert s'est cru un réformateur social appelé à une grande mission : «Tu as été choisi pour devenir le père du peuple de la ville noire!» $(V N, 105)$. Ce rôle messianique a buté sur sa vanité, sur son manque de réalisme ( $«$ je me suis cru un homme au-dessus des autres, et je n'ai pas voulu calculer ", VN, 105), enfin sur son ignorance, "piège et tourment de l'artisan qui a trop d'imagination » (VN, 109). Le compagnonnage, dans lequel Sand avait placé l'espoir que l'on sait, montre ses limites, car pour le transformer en véritable instrument d'association des travailleurs, d'après Audebert, « il faudrait un premier capital assez rond et une première pensée assez forte » $(V N, 104)$. Plusieurs personnages sandiens des romans d'avant 1848 soulignent les limites des idéologies socialistes alors répandues en France, et expriment leurs doutes. Ils gardent toutefois la certitude que si l'on n'a pas encore trouvé la clé théorique pour résoudre la question sociale, ce n'est qu'une question de temps. De ce point de vue, La Ville noire témoigne de la fin de cette assurance. Audebert est un vieil ouvrier qui a fait faillite sur toute la ligne. Sa ferme modèle l'endette, l'usine ne marche pas. Sensible et fier, il se fâche contre ceux qui ne financent pas ses projets à fond perdu. Mais quand ses affaires vont de mal en pis, il les néglige pour « rêver encore au salut du genre humain » sans toutefois jamais trouver « le secret du bonheur ni pour [s]oi ni pour les autres » $(V N, 108)$, et même pas quelque chose de « clair et d'utile à dire » quant aux « remède[s] » proposés ( VN, 106).

On a très justement souligné les traits qui rapprochent Audebert, avec ses "enthousiasmes suivis de découragements», de la figure de Pierre Leroux ${ }^{39}$, le philosophe qui a le plus influencé Sand dans sa connaissance du socialisme ${ }^{40}$. D'ailleurs, si, en 1860, l'écrivaine garde pour lui une certaine admiration - malgré le refroidissement de leurs rapports datant déjà d'avant 1848 - son détachement se confirme à la suite de l'incapacité du penseur à gérer ses projets (notamment celui relatif à l'imprimerie 
commune de Boussac). Concernant l'opinion qu'elle s'est faite de lui, on peut penser aussi à l'ouvrier de l'article du Temps déjà cité : « charlatan et ridicule », il « se pose [...] en beau parleur, en penseur éclairé », il «ne se contente plus d'être votre égal» et «veut vous faire sentir qu'il est votre supérieur» (IS, 60). Que Sand se venge des incohérences de Leroux et de la vanité présumée de l'ouvrier de 1860, n'évacue pourtant pas le problème. Car Tonine, le seul autre personnage qui, dans le roman, se préoccupe du bien-être collectif, ne professe ni ne recherche aucun système théorique pour opérer le changement. Elle est également la seule qui arrive à modifier concrètement la vie de la ville basse. Le manque de confiance de Sand n'investit pas seulement le prolétaire en tant que tel. C'est plus en général du côté de l'idéologie ouvrière que le bilan est lourd.

Sand n'épargne pas non plus ceux qui pensent pouvoir se poser en prophètes afin de débiter leurs vérités aux victimes de l'injustice sociale. Si le menuisier du Compagnon était un «apôtre " de la fraternité des travailleurs appelé directement par le Christ ${ }^{41}$, si le solide meunier d'Angibault dans le roman éponyme prophétisait - déjà beaucoup plus discrètement - la possibilité de trouver la voie pour la société égalitaire du futur ${ }^{42}$, leur dernier avatar est un ouvrier qui tient un discours confus, dénué de tout sens pratique, quelque peu mégalomane, menacé par la folie. Il est vrai que, dans son « délire », Albert de Rudostaldt prophétisait la révolution mais, bien qu'artiste et fou aux yeux du monde, il n'était nullement risible. Au contraire sa maladie pouvait devenir «une faculté exceptionnelle du genre le plus poétiquement divin", un moyen de connaissance supérieure $^{43}$. Toute autre est la situation d'Audebert. Il revient d'un banquet en son honneur - les gens de la ville haute aiment ses chansons populaires - et se voit en nouveau Pindare. Sept-Épées, de retour, le rencontre, heureux, vagabond, caricatural, ses lauriers sur la tête, accompagné d'un cortège d'enfants, les seuls destinataires possibles de ses enseignements et qui d'ailleurs le protègent plus qu'ils n'apprennent de lui quoi que ce soit.

16 Cette transformation est certes difficile à interpréter. D'une part, Audebert semble réaliser presque mot à mot, quinze ans après, la prophétie réactionnaire de l'industriel Cardonnet qui met en garde son enfant voué aux idéaux socialistes : «Tu aurais dépensé follement d'une main ce que j'aurais ramassé de l'autre; et à quarante ans, épuisé de fantaisies, à bout de génie et de confiance, [...] fou peut-être, car c'est ainsi que finissent les âmes sensibles et romanesques, [...] tu me serais revenu accablé de ton impuissance, irrité contre l'humanité, et trop vieux pour reprendre le bon chemin» $(P, \mathrm{I}, 213)$. De l'autre, si Audebert se couvre de ridicule, ses chansons sont valorisées par Tonine et par les autres comme "grandement utiles»: "C'est de la morale qui nous vient toute mâchée, [...] ça va partout, [...] [ç]a console souvent, ça apprend à voir et à sentir ce qui est beau et ce qui est bien » $(V N, 161)$. Sand ne désavoue pas la fonction et la beauté de la poésie prolétaire qu'elle a toujours soutenue, même si elle en donne ici une vision décidément plus simpliste et politiquement neutre ${ }^{44}$. D'ailleurs, la romancière restitue sa raison à Audebert au moment de lui confier l'important hyménée final : « son cœur ému avait rendu la lumière à son génie troublé » $(V N, 247)$. La dépréciation regarde moins la poésie ouvrière elle-même que l'aspiration à posséder «tous les moyens qu'il faudrait pour tirer le monde de ses peines » $(\mathrm{VN}, 247)$.

Dans l'histoire singulière d'Audebert, Sand insiste beaucoup sur sa vanité et sur son orgueil malsain, qui l'empêche d'accepter la défaite de son système boiteux et de se replier sur un programme moins ambitieux. À la fin du récit, le narrateur fournit une interprétation possible de ce que représente Audebert : «Si l'ambition de l'âme aspire à 
changer partout d'emblée la face des choses, il faut réussir ou devenir fou » $(V N, 202)$. C'est ce qui arrive en effet à Audebert, personnalité fragile, certainement, mais que Sand a ainsi choisi comme dernière incarnation des penseurs de la révolution sociale. Son instruction peu solide n'est pas la seule responsable de la vacuité de ses projets, car « de plus savants que [lui]» n'ont pas su faire mieux $(V N, 111)$. L'attente plus ou moins confiante dans les ressources des hommes de pensée pour résoudre le problème social laisse donc la place à un bilan franchement négatif. Faut-il de plus penser que Sand, convaincue depuis toujours que les travailleurs avaient en eux-mêmes les ressources pour renverser leur sort, n'attend désormais plus rien de la sagesse du peuple? Tonine, l'ouvrière plieuse, dément cette hypothèse.

\section{Une femme pour l'usine du futur}

18 Tonine est la clé de voûte de l'idéal de Sand, elle qui peut, par ses vertus, « réconcilier les contraires » des classes ${ }^{45}$ et fonder une sorte de troisième voie pour les travailleurs : fierté des origines populaires et effacement de ses aspects les plus grossiers. L'orgueil et la dignité de son identité ouvrière se conjuguent chez elle avec un esprit toujours en alerte Tonine aime lire - et des goûts raffinés. Tonine est « plus distinguée, plus instruite » que les autres ouvrières, et, dans sa pauvreté «la plus élégante, la plus soignée de sa personne» $(V N, 69)$. Désintéressée à tout enrichissement possible, charitable, elle se caractérise par son esprit d'initiative qui l'amène à répandre ses bienfaits autour d'elle. «À la fois servante et maitresse » $(V N, 165)$, Tonine a le cachet d'une sainte laïque, mais elle n'a plus rien de messianique. Solidement ancrée sur terre par son bon sens pratique, elle ne passe pas son temps à méditer sur le sort de l'humanité ou à dire la bonne parole auprès de ses concitoyens. Car, dotée d'une intelligence fine et d'une volonté de fer, bien que très douce, Tonine est une femme d'action qui trouve de suite le meilleur parti à prendre dans toute situation : elle empêche que Sept-Épées ne se brouille avec Gaucher, le sauve ensuite de la mort, remet en ordre ses livres de comptes, organise son personnel dans l'usine, calme le délire fiévreux d'Audebert, lui révèle sa véritable identité de poète, le protège de ses excès, et convainc le père Laguerre d'emménager dans une maison pleine de lumière, première image d'une transformation qui investira la ville entière. C'est elle qui donne aux petits les instruments pour bâtir leur futur: l'instruction, un travail manuel, le sentiment de sa dignité par le soin de sa personne (« elle apprenait à lire et à coudre à la petite Rose ; elle la faisait belle », VN, 165).

Rien d'étonnant, donc, si la plieuse aux mains symboliquement toujours blanches reçoit un héritage inattendu - l'usine la plus grande de la région - qui changera son sort et celui de tous les ouvriers. Si l'expédient littéraire est rodé, dans les romans des années 1840, pour escamoter le problème du financement du projet utopique, le destinataire ne l'est pas : cette fois, c'est à une femme d'ébaucher le futur heureux de l'humanité. C'est là l'aspect le plus subversif du roman, d'autant plus que cette femme est adroite et instruite autant sinon plus que le héros masculin, car elle s'est «mise au courant du mouvement industriel et commercial de la France » $(V N, 244)$ sans jamais sortir de son Val-d'Enfer, tandis que son amoureux, pour en savoir autant, a entrepris un long voyage. À défaut des certitudes politiques d'antan, le féminisme de Sand se renforce, et la dernière dépositaire des espoirs d'un avenir socialement plus juste est une femme. Sand l'a parée des vertus morales que l'on connaît déjà chez ses sœurs aînées des années 1840 - pureté, générosité, modestie, force -, sous des traits, bien sûr, qui l'en différencient. 

bâtir une nouvelle route qui assure des communications plus aisées, ainsi que de l'espace, de l'air et de la lumière à la ville noire. Mais surtout, elle transforme la " grande carcasse triste et noire » $(V N, 231)$ de la fabrique héritée en « une maison de plaisance traversée par les flots de la rivière » $(V N, 230)$, archétype d'un système économique harmonieux où le travail devient, dans la mesure du possible, un plaisir. Ensuite, elle fait preuve d'un franc-parler non exempt de dureté. À un Sept-Épées irrésolu qui ne termine pas ses phrases, Tonine rétorque brusquement «À présent quoi ?» $(V N, 139)$, et lui livre sans aucune réticence ses jugements : «Est-ce vous qui griffonnez comme ça ?... » $(V N, 137)$; « vous voilà propriétaire d'une chose qui ne vaut peut-être rien » (VN, 139). Tonine fait preuve d'un pragmatisme extrêmement efficace fondé sur un savoir concret que les héroïnes précédentes étaient loin de connaître. C'est une ouvrière, bien sûr : ni une artiste, ni une aristocrate qui se consacre à la lecture. Cela reflète peut-être des besoins nouveaux de la romancière. Dans l'effervescence sociale et intellectuelle des années 1840, quand Sand avait pleine confiance dans la force d'impact du socialisme utopique et sentait que l'on pouvait tout conquérir, les romans dessinaient leur utopie égalitaire par de longs dialogues entre les personnages qui n'approfondissaient pourtant pas les aspects concrets de cette même utopie. En tout cas, sa réalisation était reportée à plus tard. Dans la situation d'attente forcée du Second Empire dans laquelle Sand croit devoir repenser son credo politique, la création littéraire offre l'image d'un monde meilleur sans tirades idéologiques, mais décrit jusque dans les détails, et l'accomplit en un sens dans le présent de la fiction.

21 Ainsi, de retour de son voyage, Sept-Épées peut voir de ses propres yeux la fabrique où une nouvelle vie est commencée. Les salles y sont «claires, bien aérées, avec des péristyles clos et couverts " pour protéger du froid la pause des ouvriers; il ne manque pas de «bains, [...] gymnases, [...] salles d'études» $(V N, 225)$, ce qui fait de l'usine un centre vital et civique de la ville. L'exaltation de la puissance des machines ne récuse pas l'idée d'une intégration harmonieuse à la nature, car la «noble fabrique touch[e] à la campagne » et l'on "vo[it] s'ouvrir l'immense vallée avec ses noyers plantureux et ses jeunes blés inondés de lumière » $(V N, 230-231)$. Tonine opère selon les lois de la productivité - son usine "rapporte gros" $(V N, 225)$ - tout en refusant le corollaire capitaliste d'une exploitation inhumaine. Elle a trouvé la synthèse de ce qui se donnait comme dichotomique à Sept-Épées. Pour ce dernier, l'épithalame chante : « Souviens-toi du jour où tu te sentis en lutte avec ton semblable [...], en désaccord avec toi-même. Ce fut le jour où tu reconnus que, pour gagner vite, il fallait mettre l'éperon au ventre de tes ouvriers» $(V N, 250)$. Les nouveautés que Tonine inaugure dans la gestion de l'usine dessinent un véritable État social: «tous les profits sont employés à donner l'apprentissage et l'éducation gratis aux enfants de la Ville noire, des soins aux malades, des lectures et des cours aux ouvriers, des secours et des avances à ceux qui ont eu des accidents » $(V N, 225)$. Instruction gratuite, système sanitaire, caisse de prévoyance : Sand montre une conscience assez aiguë des droits sociaux à conquérir et qui coûteront encore un siècle de luttes.

On a souligné le caractère novateur et avancé de la représentation d'une autogestion ouvrière de l'usine ${ }^{46}$. Il me semble pourtant qu'il faudrait nuancer ce propos, non seulement parce que le roman brosse un contexte historique où tous les propriétaires sont d'anciens ouvriers, mais aussi parce que le problème de la propriété n'est pas résolu dans le sens d'un partage collectif. Le système voulu par Tonine n'implique pas la mise 
" en commun ", comme c'était le cas des communes agricoles au temps du Meunier et du Péché. D'où la nécessité que la volonté de Tonine soit partagée par Sept-Épées, futur mari et propriétaire : " permettez-moi de vous dire que la richesse [...] ajoutera beaucoup à votre bonheur, si vous l'entendez comme l'entend la généreuse Tonine", avance discrètement le docteur Anthime pour sonder le terrain; et Sept-Épées de la tranquilliser : « Parle ma chère Tonine, [...] ; je ne serais pas digne de partager ton sort, si je ne partageais pas tes sentiments " $\left(V N, 242^{47}\right)$. On remarque facilement les limites paternalistes d'une vision où le progrès des conditions de vie des travailleurs demeure dans les bonnes volontés des patrons, même si l'hyménée final chante que "le bonheur n'est pas dans le triomphe de la volonté isolée, mais dans l'accord des volontés conquises au bien » $(V N, 250)$, phrase que Sand aurait pu écrire vingt ans auparavant. Tonine appelle à ses côtés le fiancé ruiné, tandis que ses amis les plus chers habitent les corps de logis de l'usine. Mais on retrace mal une communauté paritaire dans ses droits et dans ses devoirs. Tonine reste apparemment la seule dépositaire du pouvoir d'employer les autres («nous sommes gagés comme surveillants de nos salles ", explique Gaucher radieux (VN, 232)). Les repas collectifs où personne ne sert sont une habitude des jours de fête, non la règle de conduite journalière. Tonine ne garde pour elle-même que le nécessaire pour vivre « aussi simplement qu'une ouvrière à son aise » $(V N, 226)$, mais apparemment il n'est pas question d'associer directement les ouvriers aux profits de l'usine, comme le proposait vainement Cardonnet fils à son père industriel.

Hors fiction, et sans trop spécifier - comme d'habitude - en 1860, Sand pense à des actes d'« association d'intérêts » entre « le producteur et l'exploiteur ", pour définir la part que l'ouvrier apporte "à la création de la richesse du patron » (IS, 62-63); en 1863 à "des individualités collectives, par de libres essais d'association, par de grandes entreprises commerciales, intellectuelles, industrielles, civilisatrices, qui ne serons pas forcées d'étouffer les petites, [...] mais qui absorberont, par leur utilité et leur succès véritables, les tentatives vaines, bizarres, irréfléchies $»^{48}$. L'irénisme de cette vision de la concurrence capitaliste a déjà trouvé une représentation narrative dans la fabrique idéale de La Ville noire, mettant à profit les capacités que Sept-Épées gaspillait dans son entreprise avortée. Il est, par contre, plus difficile d'y reconnaître un véritable «acte d'association ». Si cela a peu à voir tant avec les solutions élaborées autrefois par le socialisme, qu'avec les mesures envisagées à la même époque par le socialisme scientifique, il est indéniable que l'usine de Tonine se veut une réponse prolétaire collective au problème de l'injustice sociale et un agent de civilisation progressiste du territoire. Le profit n'y est recherché que pour être immédiatement réinvesti en bien-être collectif. Dans ce sens, dans l'évolution de la pensée politique de Sand, La Ville noire représente un moment de passage idéologique avant l'exaltation des vertus moralisatrices de la petite propriété telle que l'on la trouvera dans Nanon (1872), où la paysanne travaillera à son enrichissement pour se trouver digne de son amoureux et, heureusement bourgeoise, propriétaire d'un vaste domaine agraire, relèguera définitivement dans le passé de l'écrivaine la promotion d'un système de répartition des richesses authentiquement communautaire ${ }^{49}$. 


\section{NOTES}

1. P. Salomon, Née romancière, Grenoble, Glénat, 1993, p. 177.

2. M. Hecquet, «Sand : du socialisme à son abandon » dans D. A. Powell (éd.), Le Siècle de George Sand, Amsterdam, Rodopi, « Faux Titre », 1998, p. 61.

3. B. Hamon, George Sand et la politique "Cette vilaine chose... », Paris, L'Harmattan, 2001, p. 352.

4. À P. Viardot, 14 janvier 1859; à F. Buloz, 4 décembre 1859, G. Sand, Correspondance, Georges Lubin (éd.), t. XV, Paris, Garnier, 1981, p. 282 et 585, dorénavant $C$ suivi du numéro de tome et de la page.

5. Notice datée du 30 décembre 1851, Le Péché de Monsieur Antoine, Romagnat, De Borée, 2007, p. 49 ; ce roman dorénavant $P$ suivi du numéro de tome et de la page.

6. Bien que «moyennant l'exercice de l'éternel devoir et de l'imprescriptible droit de réclamer son perfectionnement par l'examen et la discussion ", à E. Rodrigues, 17 avril 1863, C, XVII, 585.

7. C, XVII, 581-582.

8. 28 août 1859, C, XV, 494.

9. J. Courrier, « Présentation » dans G. Sand, La Ville noire, Romagnat, de Borée, 2007, p. 44. Toutes mes citations du roman sont tirés de cette édition, dorénavant VN suivi du numéro de la page citée.

10. B. Didier, George Sand écrivain. "Un grand fleuve d'Amérique », Paris, PUF, "Écrivains », 1998, pp. 682-683.

11. Sur le rapport désormais « dépolitisé » de Sand avec Buloz, cf. T. Loué, « George Sand fut-elle un "auteur Revue des deux mondes"? Quelques remarques sur un auteur en institution (1858-1876) », dans M.-É. Thérenty (dir.), George Sand journaliste, Saint-Étienne, Publication de l'Université de Saint-Étienne, 2011, p. 76.

12. M. Hecquet, Poétique de la parabole. Les Romans socialistes de Georges Sand (1840-1845), Paris, Klincksieck, 1992.

13. É. Bordas, «Les Romans du Second Empire », dans M. Reid, M. Riot-Sarcey (éd.), George Sand, littérature et politique, Nantes, Pleins Feux, 2007, p. 125.

14. Cf. Jean de la Roche (1860), Romagnat, De Borée, 2006, p. 303.

15. Cf. Valvèdre (1861), Paris, Michel Lévy Frères, 1863, p. 12.

16. À Édouard Plouvier, 4 janvier 1860, C, XV, 645.

17. À Solange et à E. Aucante, 24 janvier 1860, C, XV, 662 et 664.

18. $C, \mathrm{XV}, 664$, Sand souligne.

19. P. Salomon, op. cit., p. 170.

20. 23 janvier $1860, C, X V, 661$.

21. 27 janvier $1860, C, X V, 670$, Sand souligne.

22. 11 février $1860, C, X V, 691$, je souligne.

23. Ce que l'on a baptisé « réalisme prophétique », I. Naginski, « George Sand mythographe », Cahier romantique, 13, Clermont-Ferrand, 2007, p. 175 sq.

24. À F. Buloz, 22 juin 1860, C, XV, 839.

25. 17 avril 1863, C, XVII, 583.

26. C, XVII, 580.

27. C, XVII, 585.

28. $C$, XVII, 589.

29. C, XVII, 555.

30. Narcisse, Paris, Michel Lévy, 1869, dorénavant $N$ suivi du numéro de page. 
31. G. Sand, Impressions et souvenirs, È. Sourian (éd.), Paris, Éditions des femmes, 2005, dorénavant IS suivi du numéro de page.

32. Notamment, sa réponse à Flaubert devient Réponse à un ami (Le Temps, 14 septembre 1871); sur toute cette question $c f$. l'indispensable A. Poli, George Sand et les années terribles, Bologna/Paris, Pàtron/Nizet, 1975, p. 229 et sq.

33. Le Compagnon du Tour de France (1841), J.-L- Cabanès (éd.), Paris, Librairie Générale Française, «Le Livre de Poche classique ", 2004, p. 152-153.

34. Sur les qualités infernales de la ville basse, $c f$. R. Bourgeois, « Ombre et lumière : l'imagination de Sand dans La Ville noire ", dans Présence de George Sand, 5, mars 1979.

35. Que l'on pense aux libéraux dans Le Compagnon, aux Bricolin dans Le Meunier d'Angibault, à Cardonnet père dans Le Péché de Monsieur Antoine.

36. Lettre non envoyée à J. Janin, $1^{\text {er }}$ octobre 1855, C XIII, 376.

37. M. Rice-De Fosse, «The Women Writer And The Worker: Social Mobility and Solidarity in La Ville Noire ", dans D. A. Powell (éd.), op. cit.

38. Journal du Voyage en Auvergne et en Velay, G. Lubin (éd.), Présence de George Sand, $\mathrm{n}^{\circ} 36$, fév. 1990 , p. 38.

39. J. Courrier, op. cit., p. 28.

40. Pierre Leroux et George Sand, Histoire d'une amitié, J.-P. Lacassagne (éd.), Klincksieck, 1973 ; Sand rencontre Leroux une dernière fois à Nohant en novembre 1859, peu avant la rédaction de La Ville noire (Ibid., p. 83).

41. Le Compagnon, op. cit., pp. 197-198.

42. Le Meunier d'Angibault, M. Caors (éd.), Paris, Les Éditions de l'Aurore, 1990, p. 145.

43. La Comptesse de Rudolstadt, L. Cellier, L. Guichard (éd.), Paris, Gallimard, "Folio Classique ", p. 555 ; cf. M.P. Rambeau, "Maladie mentale et folie dans l'œuvre de George Sand», dans E. Mosele (dir.), George Sand et son temps : hommage à Annarosa Poli, II, Genève, Slatkine/Moncalieri CIRVI, 1994.

44. Cf. «Dialogues familiers sur la poésie prolétaire ", écrits entre janvier et septembre 1842, dans G. Sand, Questions d'art et de littérature, Paris, Calman Lévy, 1878.

45. M. Besnard-Coursodon, "Particularités structurales de deux discours utopiques: Clarens et La Ville noire ", dans Présence de George Sand, 8, mai 1980, p. 22.

46. P. Vermeylen, Les Idées politiques et sociales de George Sand, Bruxelles, Éditions de l'Université de Bruxelles, 1984, p. 242 ; C. Grossir, «S'appauvrir, s'enrichir : destins romanesques croisés », dans Les Amis de Georges Sand, 33, 2011, p. 112.

47. Je souligne.

48. À É. Rodrigues, 17 avril 1863, C XVII, 591, Sand souligne.

49. E. Roy-Reverzy, «Le Discours utopique à l'épreuve du romanesque : les romans bourgeois de George Sand", dans L. Dumasy, Ch. Massol, Pamphlet, utopie, manifeste (XIX ${ }^{e}-X X^{e}$ siècles), Paris, L'Harmattan, 2001, p. 304 ; M. Hecquet, «La Fortune de Nanon, d'après l'Histoire d'un paysan ", dans Les Amis de George Sand, n 33, 2011. 
INDEX

Mots-clés : Sand (George), progressisme utopique, socialisme, Second Empire, Ville noire, Révolution (1848), développement industriel (idéalisation du), bourgeoisie et classe ouvrière (rapport entre), identité ouvrière, ambition individuelle vs perspective collective, féminisme 